\title{
Spontaneous Alternation Behavior in Human Neutrophils
}

\author{
Karen A. Selz
}

Additional information is available at the end of the chapter

http://dx.doi.org/10.5772/47851

\section{Introduction}

SAB: Spontaneous alternation behavior (SAB) generally refers to the tendency of animals, even single-celled organisms, to alternate their non-reinforced (Dember \& Richman, 1989) choices of T- or Y-maze arms on subsequent trials, following an initial trial or turn. First described over 80 years ago (Tolman, 1925), the phenomenon has been ascribed to the operation of a variety of mechanisms including Hullian reactive inhibition (Solomon, 1948), stimulus satiation (Glanzer, 1953), action decrement (Walker, 1958), curiosity (Dember and Earl, 1957), habituation to novelty (Carlton, 1969), foraging strategies (Estes and Schoeffler, 1955) and spatial working memory (Sarter, et al., 1988). Studies have suggested that the primary cue for alternation among invertebrates to be is the body turn. Vertebrates rely primarily on directional and odor cues. The fitness benefits associated with stimulus seeking and behavioral exploration, foraging, remain the most compelling explanation of why SAB is found ubiquitously and reliably (Richman, et al.1986). Although the underlying mechanism of SAB is open to study, there is general agreement that the ability to alternate choices requires that the organism remember its previous choice (Hughes, 2004).

Because SAB implicates future behavior which is statistically dependent on prior behavior and accompanied by memorially-dependant loss of degrees of freedom, SAB has been used to suggest the presence of a functional short-term memory. A left or right turn in a T- or Ymaze is a statistical function of the presence and direction of the previous turn, when such a prior turn exists.

While theories of memory are many and not the focus of this chapter, SAB is generally suggested to be recent memory dependent in animals complex enough to possess the structures postulated to underlie recent memory, and to be dependent on a more basic sensory/membrane/receptor depletion time-limited memorial mechanism in simple 
organisms and cell systems that have also demonstrated SAB. Recent memory decays, some suggest exponentially in time (Eukaszewska and Deawichowska, 1982; Lalonde, 2002). This "decay" or, alternative, "interference" assumptions are explicit in many theories of shortterm memory (e.g., Thorndike, 1914; Oberauer and Lewandowsky, 2008), so that the SAB effect would be expected to also diminish in the mean with the extension of the long leg of the T.

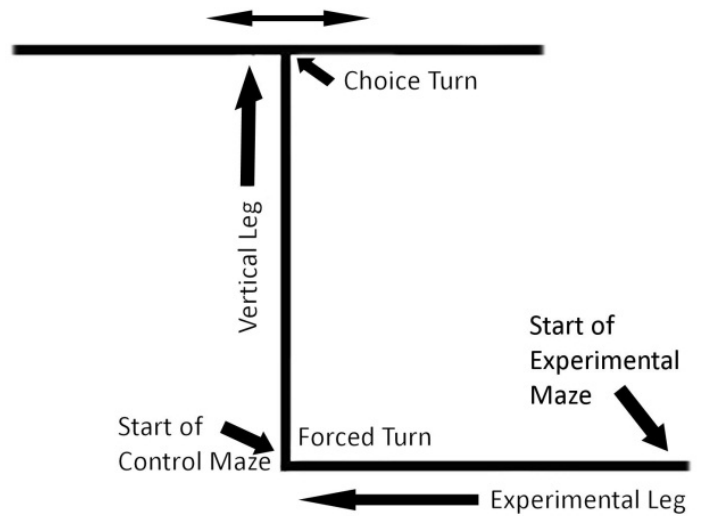

Figure 1. A general layout for T-mazes.

In fact, when the length of the vertical leg is treated as an experimental variable, using distance as a proxy for time, $\mathrm{SAB}$ consistently decreases as vertical leg length increases (Hughes, 1989). Similarly, with two-trial SAB, increasing the intertrial interval, and so the time between successive choice opportunities, has been shown to reduce alternation frequencies (Dember and Richman, 1989). This inverse relationship follows from a memorybased view of $\mathrm{SAB}$, has been used to suggest that the longer the distance between successive turns, the greater the probability that the organism will "forget" the direction of its previous turn and choice will return to a statistically equiprobable condition (Hughes, 1989). No particular mechanism of "memory" is implied in this context. That is, any mechanism that causes current actions to be influenced by previous actions could potentially lead to SAB in general and to the loss of SAB with increased distance/time between the forced and choice turns.

PMN: Human polymorphonuclear leucocytes, PMN, are highly motile cells averaging 12 to $15 \mu \mathrm{m}$ in diameter, exhibiting prominent, lobular nuclei. They emerge from bone marrow stem cells and are the most populous of the white blood cell category. PMNs are essential for host defense participating in both acute and chronic inflammatory processes. Other related properties include the regulation of immune responses, angiogenesis, and in physical interactions with neoplasms. Making this wide variety of functions possible is their dynamically adaptive behavior exemplified by the range of intrinsic patterns of motility. Following my recent work demonstrating phase transition scenarios in PMN morphodynamics (Selz, 2011), I developed a new method to assess the autonomous behavior of these human PMNs and whether single constituent cells exhibit SAB. 
Recall that whereas the natural physiological environment for autonomous motion in human neutrophils contains the PMN's endogenous tropic peptide attractant, the classical "spontaneous alteration", SAB design of these studies requires that the observed behavior, the cell's spontaneous motion, be unperturbed. The necessary absence of these chemoattractants, leads to a multitude of required trials for the acquisition of a single data point. It was for this reason that I recorded hundreds of PMNs, increasing the number of trials until $n>50$ left/right decisions at the T-intersections in each of the three types of mazes will have been observed.

\section{Methods and procedures}

Three similar T-mazes were developed; 1) a control T-maze, 2) a forced-turn T-maze, and 3) a long-leg forced turn T-maze. As SAB is the tendency of an organism to alternate successively turning left and then right in a maze, the control maze is the one in which the first turn is the only turn. Cells in control mazes are expected to turn to the right or left with roughly equal probability. However, in the second, forced turn maze, in which the cell must always make a right turn before reaching the experimental left versus right choice point of the $\mathrm{T}$, the statistical expectation is that more cells will turn left at the T. In the third maze, the $\mathrm{SAB}$ effect can decay in the mean with the extension of the long leg of the $\mathrm{T}$.

The mazes were constructed on standard glass slides, inside a $12 \mathrm{~mm}$ diameter, $25 \mu \mathrm{m}$ deep well. The specifications are indicated in the figures 2. Figure 3 is an electron micrograph of a set of the nano-structured mazes on a finished slide. Tolerances were $\pm 5 \%$. Sidewall angles of mazes were $88-90^{\circ}$. Surface variation (roughness) was in the neighborhood of $10 \mathrm{~nm}$.

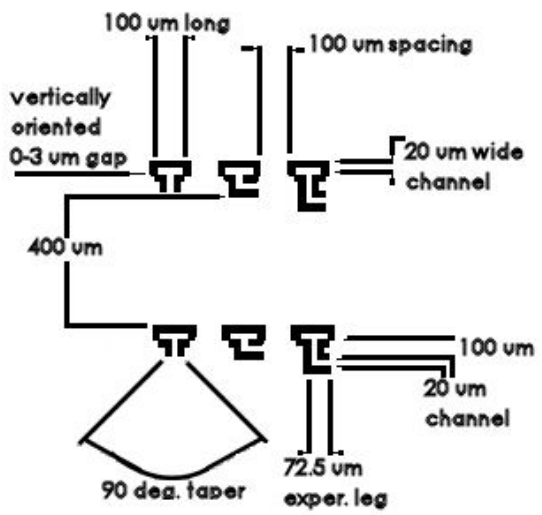

Figure 2. A rough schematic of the mazes on each slide. 


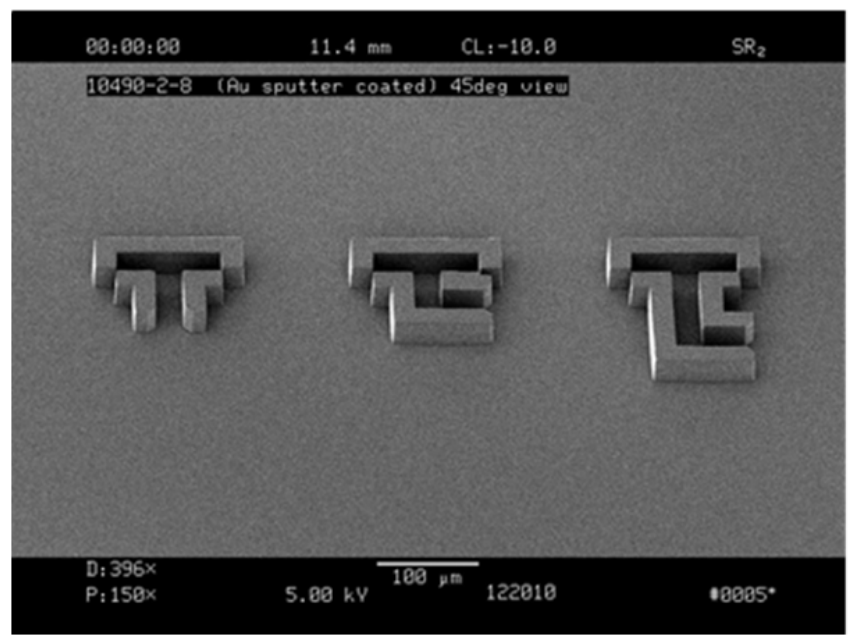

Figure 3. An EM image of one set of mazes on a slide. Note the taper at the entrance of each maze and the flow-through gap at the terminal ends of the mazes (0um at the base to 3um at the top of the maze walls). These allow the passage of medium, but not the entry or exit of PMN.

After gentle sedimentation, PMNs (along with some other white cells and platelets) were removed from the buffy coat by micropipette and, along with associated plasma. The $5.7 \mu \mathrm{m}$ gap of standard slides and coverslips is smaller than the average diameter of PMNs, leading to some mechanical compression of the cells and contributing to their activation, as well as allowing the cell to move along the slide substrate and cover slip simultaneously (Malawista and deBoisfleury Chevance, 1997). The slides used in this study do not suffer from these deficits. All samples were drawn from 5 healthy, adult volunteers (3F, 2M, ages 25-47). All slides were pre-filled with Lactated Ringer's solution, that is isotonic with blood. This was done to avoid cells being deposited into the maze ends, as well as to avoid trapped air in the mazes, and to dilute the PMN sample.

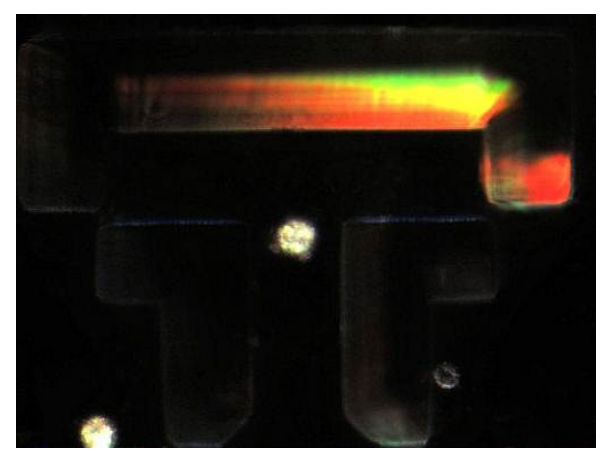

Figure 4. Shows a typical trial, in which a PMN (with evident lobular nuclei) translocates up the vertical leg of a control T-maze while another lingers outside the maze. 
As implied by the word "spontaneous," SAB studies require that the cell's motion be observed without manipulating exogenous agents. Lactated Ringer's solution provided a uniform, "undoped" medium base. Without the activating influence of chemoattractants or physical activators (e.g. heat, pressure, sheer), many trials were required for the acquisition of each data point. In total, 102 PMN navigated the control maze past the decision point, 57 navigated the forced-turn maze, and 82 PMN completed the long-legged forced-turn Tmaze.

PMN autonomous motions were observed through an Olympus BX41 microscope with a mixed CytoViva dark field and fluorescent optical illumination system. This incorporates a high-aperture, cardioid annular condenser (www.scitech.com.au) unique to this system and which makes possible visualization of objects below $90 \mathrm{~nm}$ in diameter in real time, including cellular samples in an unfixed, living, active state (Samoylov, et al., 2005; Vainrub, et al. 2006). Because PMNs were treated gently, avoiding perturbations of column separation and elution, it became possible to reliably study a PMN continuously for extended times without the onset of granular clumping, membrane blebbing and other signs of impending apoptosis (Lodish, 2005). Whenever a PMN moved to the left or right beyond the choice point of a maze, its choice was recorded.

\section{Results}

Left-Right choices in the control maze were not significantly different from equiprobability (Left $=49.02 \%$; Right $=50.98 \% ; \chi^{2}(1)=0.039, \varrho=0.843$ ). However, in the forced-turn T-maze, $63.16 \%$ of the cells that had been forced to turn Right previously, turned Left at the decision point $\left(\chi^{2}(1)=3.947, \varrho=0.0469\right)$. Contrary to initial expectation of the disappearance of the SAB effect with the increased length of the vertical leg of the maze, this effect persisted and even appeared to be strengthened in the long-legged forced-turn maze, in which $67.07 \%\left(\chi^{2}(1)=9.561, \varrho=0.0021\right)$ of completing cells turned Left at the decision point, long after a forced Right turn.

\section{Conclusions}

This is the first demonstration that $\mathrm{SAB}$ is present in PMN, a constituent cell of the human body. While SAB has been shown in human spermatozoa (Brugger, et. al., 2002), sperm cells are required to function in the world exterior to the body, while PMN are not.

The apparent strengthening of SAB in the long-legged forced-turn T-maze compared to the standard forced-turn maze, may be partially attributable to the higher $\mathrm{n}$ in the former and natural variation in data, but observation of hundreds of PMN in the maze environments suggests it might also result from a sort of practice effect of "frustrated" turn attempts in the channel, in a dose-response like paradigm. Length of time/distance spent in foiled attempts to turn may then serve as an order parameter. The persistence of SAB in this condition may also suggest multiple time scale, memory-like mechanisms operating in PMN. 
There are, in fact, established physiological mechanisms and behavior that are consistent with this speculation and with my qualitative microscopic. PMNs are known to oscillate on multiple temporal and spatial scales, from $7 \mathrm{sec}, 70 \mathrm{sec}$, and $260 \mathrm{sec}$ membrane potential fluctuations (Jäger, et al., 1988) and 25sec calcium flux oscillations, to the 28 sec G-F-actin oscillations (Marks and Maxfield, 1990), to 21.6sec and 230sec glycolytic cycles that produce $\mathrm{NAD}(\mathrm{P}) \mathrm{H}$ oscillations (Jäger, et al., 1988), and 10sec and 20sec pericellular proteolysis fluctuations (Marks and Maxfield, 1990), among many others. The time series in my recent study of PMN morphodynamics demonstrated scaling, board band power spectra with multiple resonances, suggesting a constellation of motility times (Selz, 2011). While there is debate over the specific fitness value(s) and mechanism(s) underlying SAB, it is clearly a phylogentically highly conserved behavior (Richman, et al.1986), and so, is assumed to be valuable. It follows that multiple mechanistically diverse time scales of function could be recruited to its service. This suggests the possibility of decentralized control in a highly interconnected, living dynamical system through local feedback. The necessary and sufficient conditions for a stable macro-system composed of multiple smaller systems operating on a variety of temporal and spatial scales are known in non-biological contexts (Ramakrishna and Viswanadham, 1982). Work is currently underway, adapting these constraints to a PMN model.

Because, with the prior forced turn, systems statistically deviate from equiprobability in a later choice circumstance, SAB represents a reduction in population behavioral entropy, and a situation in which a reduction in the behavior degrees of freedom and reduced statistical behavioral entropy is favored evolutionarily. This finding is contrary to some findings of increased entropic states being associated with greater biological health and/or function (e.g. Mandell, 1987; Paulus et al., 1980).

\section{Author details}

Karen A. Selz

Franklin-Fetzer Laboratory, Cielo Institute, Asheville, NC, USA

\section{References}

Brugger, P, Macas, E., and Ihlemann, J. (2002) Do sperm remember? Behavl Brain Res., 136(1), 325-8.

Carlton PL. (1969). Brain-acetylcholine and inhibition. In: Tapp JT, editor. Reinforcement and behavior. New York: Academic Press,p. 286-327.

Dember WN, Earl RW.(1957). Analysis of exploratory, manipulatory and curiosity behaviors.Psychol Rev, 64:91-6.

Dember, W. N., \& Richman, C. L. (Eds.) (1989). Spontaneous alternation behavior. New York: Springer. 
Estes WK, Schoeffler MS. (1955). Analysis of variables influencing alternation after forced trials. J Comp PhysiolPsychol, 48:357-62.

Eukaszewska, I., Deawichowska, (1982). How long do rats remember the spatial arrangement of visual stimuli? ActaNeurobiol.Exp, 42, 127-133.;

Glanzer M. (1953). Stimulus satiation: an explanation of spontaneous alternation and related phenomena. Psychol Rev, 60:257-68.

Hughes, R. N. (1989). Phylogenetic comparisons. In W. N. Dember \& C. L. Richman (Eds.), Spontaneous alternation behavior (pp. 38-57). NewYork: Springer.

Hughes, R. N. (2004). The value of spontaneous alternation behavior (SAB) as a test of retention in pharmacological investigations of memory. Neuroscience $\mathcal{E}$ Biobehavioral Reviews, 28, 497-505.

Jäger U, Gruler H, Bültmann B (1988) Morphological changes and membrane potential of human granulocytes under influence of chemotactic peptide and/or echo-virus type 9. Klin Wochenschr 66: 434.

Lalonde, R. (2002). The neurobiological basis of spontaneous alternation, NeurosciEBiobehav Rev, 26(1), 91-104.;

Lodish HF (2005) Molecular and Cell Biology. W.H. Freeman, New York.

Malawista SE, deBoisfleury Chevance A (1997) Random locomotion and chemotaxis of human blood polymorphonuclear leukocytes (PMN) in the presence of EDTA: PMN in close quarters require neither leukocyte integrins nor external divalent cations. PNAS 94:11577-82.

Mandell AJ (1987) Dynamical complexity and pathological order in the cardiac monitoring problem Physica D 27 235-42.

Marks PW, Maxfield FR (1990) Local and global changes in cytosolic free calcium in neutrophils during chemotaxis and phagocytosis. J Cell Biol 110:43.

Oberauer K, Lewandowsky S (2008) Forgetting in immediate serial recall: decay, temporal distinctiveness, or interference? Psychology review, 115(3), 544-576.

Paulus MP, Geyer MA, Gold LH, Mandell AJ (1990) Application of entropy measures derived from the ergodic theory of dynamical systems to rat locomotor behavior. Proc.Natl.Acad.Sci. 87: 723-727.

Ramakrishna A, Viswanadham N (1982) Decentralized Control of Interconnected Dynamical Systems. IEEE Transactions on Automatic Control, 27(1), 159-164.

Richman, R., et al.(1986). Spontaneous alternation behavior in animals: A review. Current Psychology, 5(4), 358-91.

Samoylov AM, Samoylova TI, Pustovyy OM, Samoylov AA, Toivio-Kinnucan MA, et al. (2005) Novel metal clusters isolated from blood are lethal to cancer cells. Cells Tissues Organs 179:115-124.

Sarter M, Bodewitz G, Stephens DN. (1988). Attenuation of scopolamine induced impairment of spontaneous alternation behaviour by antagonist but not inverse agonist and agonist beta-carbolines. Psychopharmacology, 94:491-5.

Selz KA, (2011).A Third Measure-Metastable State in the Dynamics of Spontaneous Shape Change in Healthy Human's White Cells.PLoSComputBiol 7(4): e1001117. doi:10.1371. 
Solomon RL. (1948). The influence of work on behavior. Psychol Bull;45:1-40.502; R.N. Hughes (2004)Neuroscience and Biobehavioral Reviews 28, 497-505

Thorndike EL, The Psychology of Learning, N. Y., Teachers College, 1914

Tolman EC. (1925). Purpose and cognition: the determiners of animal learning. Psychol Rev, 32:285-97.

Vainrub A, Pustovyy O, Vodyanoy V (2006) Resolution of $90 \mathrm{~nm}$ (lambda/5) in an optical transmission microscope with an annular condenser. Optics Lett 31, 2855-2857

Walker EL. (1958). Action decrement and its relation to learning. Psychol Rev, 65:129-42. 